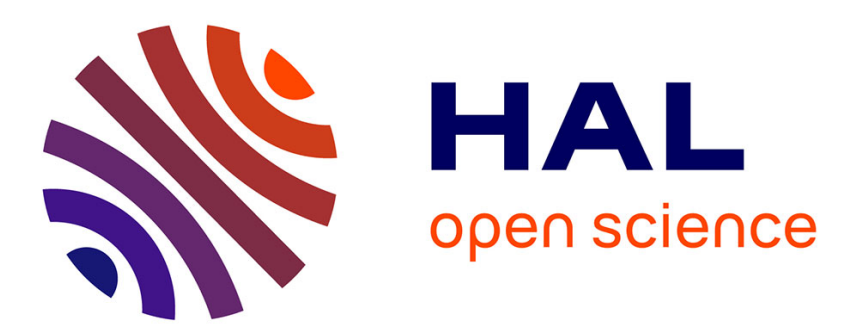

\title{
Gérer les risques au travail: place et rôle du droit dans le domaine des risques sanitaires. Présentation du dossier
}

\author{
Éric Drais, Jérôme Pélisse
}

\section{To cite this version:}

Éric Drais, Jérôme Pélisse. Gérer les risques au travail: place et rôle du droit dans le domaine des risques sanitaires. Présentation du dossier. Droit et Société, 2017, 2 (96), pp.235-240. $10.3917 /$ drs.096.0235 . hal-03565597

HAL Id: hal-03565597

https://hal-sciencespo.archives-ouvertes.fr/hal-03565597

Submitted on 11 Feb 2022

HAL is a multi-disciplinary open access archive for the deposit and dissemination of scientific research documents, whether they are published or not. The documents may come from teaching and research institutions in France or abroad, or from public or private research centers.
L'archive ouverte pluridisciplinaire HAL, est destinée au dépôt et à la diffusion de documents scientifiques de niveau recherche, publiés ou non, émanant des établissements d'enseignement et de recherche français ou étrangers, des laboratoires publics ou privés.

\section{()ㅜ) $\Theta$}

Distributed under a Creative Commons Attribution - NoDerivatives| 4.0 International 


\title{
Gérer les risques au travail : place et rôle du droit dans le domaine des risques sanitaires. Présentation du dossier
}

\author{
Éric Drais *, Jérôme Pélisse ** \\ * Institut National de Recherche et de Sécurité au Travail (INRS), 1 rue du Morvan, CS 60027, F-54519 Vandœuvre-lès-Nancy. \\ <eric.drais@inrs.fr> \\ ${ }^{* *}$ Centre de Sociologie des Organisations (CSO), Institut d'études politiques de Paris, 9 rue Amélie, F-75007 Paris. \\ <jerome.pelisse@sciencespo.fr>
}

In: Droit et Société, 2017/2, №96, p. 235-240

Les politiques de santé et sécurité au travail ont fait l'objet d'importantes transformations depuis les années 1990-2000, en particulier en France. Elles bénéficient aujourd'hui d'une imposante instrumentation juridique qui structure la gestion des risques ; c'est-à-dire l'identification, l'évaluation, l'analyse et la prévention des risques, ainsi que leur réparation. Cette dernière a été historiquement centrale dans la construction d'un droit du travail spécifique et distinct du droit civil, ainsi que dans le développement d'un État-providence. Le droit du travail, mais aussi celui de la sécurité sociale et plus généralement de la santé, ainsi que le droit de l'environnement sont cependant confrontés à des mutations technologiques et organisationnelles majeures, qui pèsent également sur la transformation du travail et de ses risques dans un contexte de forte valorisation sociale de la santé.

Quelle place occupe alors le droit? Quel rôle joue-t-il dans les régulations qui se constituent autour des risques professionnels ? C'est l'objet de ce dossier qui entend offrir une mise en perspective des usages du droit à partir de différentes recherches actuellement menées en France comme à l'étranger sur des problèmes de santé ou de sécurité que rencontrent des travailleurs et que doivent gérer des organisations publiques et privées compte tenu de leurs responsabilités. Le droit représente un point d'entrée particulièrement intéressant pour comprendre l'accroissement de la complexité du travail et des modèles de risques comme les difficultés de la prise en charge de la santé-sécurité et de la prévention. Le droit constitue, en effet, autant un cadre contraignant qu'une ressource, un registre normatif porteur de valeurs et de principes constituant des appuis pour l'action, autant qu'un horizon susceptible de peser sur les organisations et les acteurs qui ont en responsabilité, et/ou qui gèrent directement, ces risques collectifs.

Considérant que le droit ne s'impose pas mécaniquement, mais offre des occasions favorables pour définir les situations et les actions, il nous intéressait de rassembler des travaux aussi bien monographiques que comparatifs, documentant des cultures juridiques diverses à l'égard des risques dans des univers professionnels variés, par l'intermédiaire d'enquêtes sociologiques et d'études menées dans le 
cadre de recherches-action. Ce dossier permet d'aborder l'intégralité du cycle de construction du risque; de sa connaissance à sa reconnaissance et sa prévention. Les configurations proposées vont du traitement des alertes et de la fabrication des problèmes sanitaires ou sécuritaires à la mise en œuvre des règles de droit visant à prévenir les risques. Ces règles sont activées au quotidien par les employeurs et travailleurs eux-mêmes ou parfois avec l'intervention d'acteurs tiers (inspecteurs en particulier, mais aussi psychologues, médecins ou consultants). Si les règles de droit orientent la conception de la santé et des urgences, la complexité des questions de santé et de sécurité au travail met à mal la matière juridique et ses spécialisations. C'est pourquoi ce sont les usages du droit (ou plutôt des droits, car ils peuvent être multiples), par les salariés et bien souvent par des intermédiaires - et non des professionnels du droit -, qui sont au centre de ce dossier.

Les travaux réunis ici analysent les défis posés par l'exposition à des risques autant anciens qu'émergents (liés au BTP ou aux pesticides comme aux nanomatériaux et aux risques psychosociaux) et l'introduction de démarches de prévention sinon de gestion des risques dans des univers qui n'en sont pas nécessairement familiers (comme les laboratoires scientifiques ou les travailleurs indépendants). La mise en regard de ces différents risques, dans le domaine du travail principalement - mais la comparaison intègre aussi un cas sur les risques pour l'environnement et la sécurité sanitaire des aliments -, permet de souligner des convergences fortes et des apports significatifs à la mise en commun de ces articles. En premier lieu, considérer les différents matériaux juridiques de la santé-sécurité au travail permet d'aller du droit positif (droit du travail, de l'environnement, etc.) à la normativité la plus spontanée et d'analyser comment ces registres se complètent ou se combinent en situation. Cela donne à voir une transformation du statut du droit et de son rapport à l'action publique. En second lieu, la mise en risque implique le plus souvent une métrologie et une instrumentation gestionnaire, mais aussi une anticipation des possibles et la délimitation juridique d'obligations et de responsabilités. De ce point de vue, un déplacement général est à souligner, qui conduit à passer d'une obligation de moyens qui doivent être mis en œuvre, à une obligation de résultat. Les employeurs sont désormais systématiquement tenus par ces derniers et les juridictions peuvent les évaluer potentiellement en aval. Si cette transformation des règles de droit témoigne d'une genèse sociale européenne, elle constitue une matrice commune aux secteurs et activités étudiés - les cas brésiliens et américains inclus dans ce dossier montrant que des logiques communes de gestion des risques professionnels peuvent être identifiées même lorsque les règles de droit national sont éminemment différentes. Ensuite, un troisième intérêt réside dans la reconnaissance de l'importance de l'expertise (bien problématisée depuis une dizaine d'années) dans la gouvernance des risques. Le dossier évoque la place des savoirs et leur articulation avec le droit dans le domaine du travail, quelle que soit l'institutionnalisation de cette expertise en matière de risque sanitaire (de l'expertise CHSCT, très cadrée juridiquement, aux situations plus floues, y compris de recherche-action, n'impliquant a priori pas le droit). Enfin, un quatrième apport de ce dossier repose sur l'analyse des activités et des statuts de ces experts responsables de ces processus d'évaluation et de 
gestion des risques (au premier rang desquels figurent les inspecteurs du travail), qui ont à traduire et à faire appliquer les règles de droit en matière de gestion des risques. D'autres acteurs proches comme les cadres administratifs d'agences sanitaires ou de ministère, ou les ingénieurs de sécurité, même s'ils sont bien moins dotés en ressources juridiques, interviennent aussi. Évidemment, des salariés, des chercheurs (des directeurs de laboratoire aux doctorants), des directions et cadres d'entreprise ou représentants du personnel, interviennent aussi comme des figures d'intermédiaires du droit et, sans en être des professionnels, s'y confrontent et l'utilisent au quotidien dans leurs activités.

Les deux premiers textes qui composent ce dossier analysent ainsi la place et le rôle du droit dans des processus d'alerte en matière de risques. Dans le premier cas, Jean-Noël Jouzel et Giovanni Prete documentent l'exposition aux pesticides dans l'agriculture et «la domestication de l'alerte EPI» dont ils retracent l'histoire et la circulation dans différentes arènes au cours des années 2000. Décrivant une succession d'intéressements, ils soulignent ce que ces opérations doivent au droit, en particulier administratif, qui permet de rendre les alertes audibles pour les décideurs. Celui-ci conduit littéralement à les normaliser, quitte à en modifier substantiellement le contenu, la forme ou les enjeux. Mireille Lapoire-Chasset vérifie également l'importance de l'énonciation du droit pour la prise en charge des risques dits "psychosociaux». En s'appuyant sur des retours d'expériences, elle analyse comment les interventions de nombreux experts ou consultants, formés à des disciplines et des approches variées, souvent critiques à l'égard de la réglementation (psychodynamique du travail, clinique ou ergonomie de l'activité), s'appuient sur le droit pour engager un débat sur le travail et favoriser la prévention. L'argumentation juridique participe, en effet, autant à l'identité d'action des préventeurs qu'à la construction de la santé des salariés.

Entre l'alerte et le règlement des risques, intervient parfois le temps de l'inspection responsable du contrôle de la réglementation. Les deux articles suivants montrent des inspecteurs en prise, d'une part aux évolutions du droit, mais aussi aux transformations des entreprises et des risques. Dans le cas brésilien, Roberto Pirès analyse comment, en étant arrimés à deux registres d'action identifiés parmi les pratiques des inspecteurs du travail, les règles de droit - outils autant qu'objectifs à faire respecter - permettent de gagner en efficacité pour gérer des risques variés en matière d'accident du travail en lien avec les questions d'emploi ou de fiscalité. De leur côté, Olivier Borraz, Ivanne Merle et Mara Wesseling, en comparant en France les inspecteurs du travail, à ceux des installations classées et de sécurité sanitaire des aliments, font apparaître non seulement des évolutions voisines en matière de procéduralisation et de rationalisation des inspections, mais aussi des réactions professionnelles différentes, qui s'expriment sous la forme du refus, de la codification ou de la mesure du risque. Ces stratégies signent cependant une volonté commune de préserver des marges de manœuvre et que soit respectée leur autonomie professionnelle dans la traduction de la réglementation, ce qui contraste avec leur souhait de limiter leurs responsabilités. 
Les deux derniers articles du dossier portent sur l'émergence et la prise en charge par les organisations d'un même type de risques : celui lié aux nanoparticules et nanomatériaux élaborés par des laboratoires de recherche ou produits par des entreprises technologiquement innovantes. Éric Drais montre ainsi comment, en France, le principe de précaution constitutionnalisé en 2005 s'hybride avec les principes de prévention, instaurés de longue date. Basé sur des études de cas, l'article présente des configurations où les salariés ou leurs représentants activent la prévention en faisant appel à la précaution, mais également se servent des règles de prévention pour alimenter la précaution! Cette articulation en matière d'information comme de gestion des risques signe une convergence originale entre le droit du travail et celui de l'environnement pour les risques professionnels émergents. Enfin, en s'appuyant sur une enquête ethnographique, Jérôme Pélisse s'est intéressé à la gestion quotidienne des risques dans des laboratoires français et américains de nanosciences. Les tensions entre science et droit, entre innovations et prises de risque et entre réglementation et prévention, alimentent de vives négociations. Une forme de juridicisation de la recherche est repérable dans les différents laboratoires : l'analyse des acteurs et de leurs pratiques, qu'ils soient chercheurs, mais aussi ingénieurs "de terrain " ou ingénieurs "de papier " montre à nouveau l'importance des intermédiaires du droit pour comprendre les manières dont, à partir ou autour du droit, se gèrent les risques en situation professionnelle.

Le droit constitue ici un analyseur pertinent, et à plusieurs égards, est source de renouvellement pour les chercheurs qui ont participé à ce dossier, et à la journée d'étude qui l'a précédé. Celle-ci était organisée par deux institutions qui abritent une longue tradition d'étude des risques et partagent une même attention pour la dimension juridique de l'expertise : le Centre de sociologie des organisations d'une part, et l'Institut national de recherche et de sécurité d'autre part, avec une même attention pour la dimension juridique de l'expertise. Moyen et instrument, autant qu'horizon d'action et ensemble de règles fixant des repères pour l'action et des responsabilités, le droit constitue bien une entrée heuristique pour saisir la complexité grandissante de nos organisations et de nos activités ainsi que les risques qu'elles affrontent, en particulier dans ces contextes structurants que sont les pratiques de travail. Dans l'écheveau des normativités juridico-scientifiques liées aux questions sanitaires au travail, le droit est un moyen d'éclairer la genèse, le fonctionnement et la portée des dispositifs de prévention. Le droit n'est pas pour autant le seul instrument d'action, et le dossier permet surtout, finalement, de comprendre comment il s'articule avec d'autres dispositifs (techniques, organisationnels, gestionnaires) et d'autres registres (professionnels, économiques, sociaux) pour contribuer à la construction de cultures de sécurité et de formes de légalité, à la fois locales et reliées aux corpus et aux textes qu'utilisent et auxquels se réfèrent plus ou moins directement des figures variées d'intermédiaires du droit. 


\section{- Les auteurs}

Éric Drais est sociologue au laboratoire Gestion et Organisation pour la Santé Sécurité au Travail, à l'INRS (Institut National de Recherche et de Sécurité au Travail). Ses travaux portent sur la gestion et le management de la santé-sécurité (norme ISO 45001, etc.) et l'organisation de la prévention, en particulier à l'égard des nanomatériaux. Il a participé à plusieurs projets nationaux ou internationaux sur ce sujet (OSHA Europa, ANR Nanonorma, etc.). Il oriente actuellement ses recherches vers une sociologie du travail de prévention, un travail de traduction et d'articulation des règles de santé et de sécurité à l'ombre du droit.

Jérôme Pélisse, professeur de sociologie à Sciences Po et chercheur au CSO (UMR, CNRS), étudie divers objets au croisement de la sociologie du travail et de la sociologie du droit; notamment les transformations des relations professionnelles en entreprise, les régulations du temps et celles de la santé - sécurité au travail. Il a aussi mené des recherches sur les interactions entre science et droit au prisme des situations d'expertise judiciaire ou de la gestion des risques dans les laboratoires scientifiques. Il a notamment publié :

- Droit et régulations des activités économiques : perspectives sociologiques et institutionnalistes, Paris : LGDJ Lextenso, coll. « Droit et Société », 2011. 